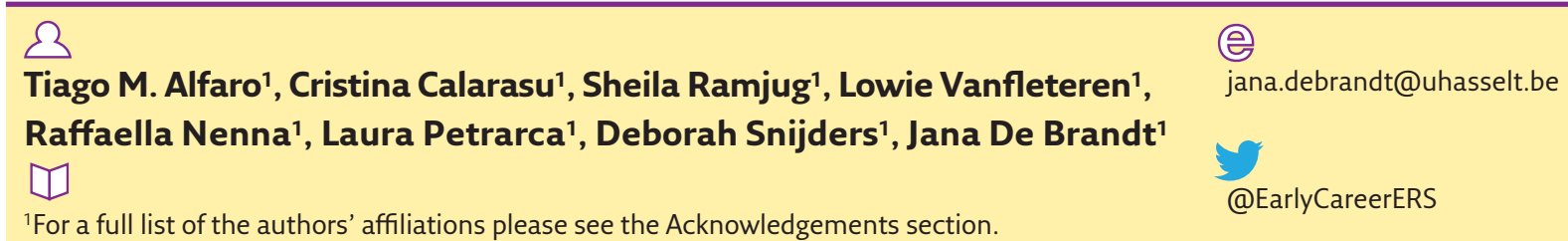

\title{
New members of the ECMC and lessons from the ATS Conference
}

\section{Early career forum}

\section{New members of the ECMC}

The Early Career Member Committee (ECMC) has four new members who will official start their mandate at the ERS International Congress 2018 in Paris, France. The new committee members are Lowie Vanfleteren (Assembly 1), Cristina Calarasu (Assembly 10), Tiago Alfaro (Assembly 12) and Sheila Ramjug (Assembly 13).

\section{Lowie Vanfleteren, Assembly 1}

Lowie Vanfleteren is the new early career member (ECM) representative of Assembly 1 (Clinical). He is a pulmonologist and senior lecturer in pulmonary medicine at Sahlgrenska University and Sahlgrenska University Medical Hospital, Gothenburg, Sweden, and is also affiliated with CIRO+, a centre of expertise in chronic organ failure, in Horn, the Netherlands, and the Dept of Respiratory Medicine at Maastricht University Medical Center in Maastricht, the Netherlands. In 2015, he obtained his PhD on "COPD and comorbidities" at Maastricht University. His clinical and scientific interests are (complex) COPD and its comorbidities, pharmacological management of COPD, bronchoscopic lung volume reduction, pulmonary rehabilitation, managed care, and severe asthma. $\mathrm{He}$ is an associate editor of Respiration.

\section{Cristina Calarasu, Assembly 10}

Cristina Calarasu is the new ECM representative of Assembly 10 (Respiratory Infections). She is a pulmonologist working for a private clinic in Craiova, Romania, and a Teaching Assistant for Phthisiology at the University of Medicine and Pharmacy of Craiova. She is the cofounder of the 9G Section (Next Generation), which is the youngest section of the Romanian Pneumology Society (SRP). Her PhD thesis looks at "Clinical and epidemiological links between TB and HIV between 2005 and 2015 in Southern Romania". She is also an investigator involved in clinical studies (asthma, COPD, pneumonia and bronchiectasis). She graduated in Integrative Psychotherapy taught by the Romanian Association for Integrative Psychotherapy, and believes communicating well with patients and changing behaviour leads to better compliance and to stronger benefits.

\section{Tiago Alfaro, Assembly 12}

Tiago Alfaro is the new ECM representative from the recently created Assembly 12 (Interstitial Lung Diseases). He works as a pulmonologist with clinical and research interests in interstitial lung disease (ILD) at Centro Hospitalar e Universitário de Coimbra, Coimbra, Portugal, where he is also in
Cite as: Alfaro TM, Calarasu C, Ramjug S, et al. New members of the ECMC and lessons from the ATS Conference. Breathe 2018; 14: 229-231. 
charge of the Respiratory Medicine Dept Research Laboratory. His PhD studies were based on the effects of caffeine and adenosine receptors on respiratory disease. He is an associate editor of the Pulmonology Journal (previously Revista Portuguesa de Pneumologia) and a member of the National Board of Pulmonology of the Portuguese Medical Association.

\section{Sheila Ramjug, Assembly 13}

Sheila Ramjug is the new ECM representative from the recently created Assembly 13 (Pulmonary Vascular Diseases). She is a respiratory registrar in her penultimate year of training at Manchester Foundation Trust, Manchester, UK, with a specialist interests in pulmonary vascular diseases, pulmonary transplantation and ILD. She has dual accreditation in medicine and surgery, having previously trained in cardiothoracic transplantation. For her Doctor of Medicine research under the supervision of Robin Condliffe (University of Sheffield and Northern General Hospital, Sheffield, UK), she studied the effects of pulmonary arterial hypertension in connective tissue disease and adult congenital heart disease. She is excited that pulmonary vascular diseases now have a formal platform within the ERS and looks forwards to promoting this growing field.

\section{Learning from our colleagues from the American Thoracic Society}

This year, the American Thoracic Society (ATS) annual meeting was held in San Diego, CA, USA (May 18-23, 2018). It marked the 114th year of the conference, making it the longest running large-scale conference in the world offering milestone research in pulmonary, critical care and sleep medicine. This year, in $~ 6700$ abstracts and 500 sessions, $>800$ presenters shared their latest research findings. It was also a wonderful networking opportunity for researchers with a specific interest in pulmonary medicine from all over the world.

Here, we describe a new type of poster discussion with an example and a session of an Interest Group to show how the ATS Conference is constantly evolving.

\section{RAPiD session}

The ATS Conference 2018 offered a new and more dynamic opportunity to exchange ideas between the presenters in a poster discussion session called RAPiD (Rapid Abstract Poster Discussion). The entire session was grouped in themes according to the research field and each presenting author was first invited to talk about their main results of their work in a 90-s presentation, using a microphone and with the support of a single PowerPoint slide. After this quick presentation, each presenter was asked to stand by their poster to reply to possible questions or to clarify points of their research. The third part of the session consisted of a general discussion mediated by the moderators with the aim of answering specific questions from the presenters of related abstracts, trying to extract a take-home message from the most recent evidence.

For example, a RAPiD session entitled "Pediatric viral airways disease: mechanisms and clinical impact" explored the interaction between host and viruses. The first group of presentations focused on the effect of a virus on cells; in particular, they investigated the role of the respiratory syncytial virus (RSV) on regulation of $\beta_{2}$-adrenergic or glucocorticoid receptors, hyaluronan-dependent leukocyte adhesion molecules, tight junction proteins, and alveolar-like macrophages. The second theme was about the clinical aspects of bronchiolitis, such as response to therapies and different phenotypes of the disease, according to age, adaptive immune response and type of virus. The third theme focused on the role of antibiotics in the treatment and prevention of respiratory tract infections.

During the discussion at the end of the RAPiD session, several authors expressed their point of view regarding the virus' effect on the host. Some researchers in particular strongly argued that the virus is the major factor responsible for the clinical and immunological phenotypes in infants, whether other presenters expressed the opinion that it would be the interaction between the host and the virus that is important for the manifestation of the disease.

It was an amazing experience because it gave the opportunity to all the presenting authors to share personal opinion and results, providing new input for future work.

\section{Interest Group in Children's Interstitial and Diffuse Lung Disease Research Network}

This year, there was the first Interest Group on the Children's Interstitial and Diffuse Lung Disease Research Network (chILDREN). An Interest Group of the ATS is defined as a community of ATS members (typically between 15 and 50 persons) and attendees at the ATS Conference with an interest in networking and advancing a specific area of knowledge, healthcare, a research initiative or technology within the areas of pulmonary, critical care and sleep medicine.

This group is working to set up and create a network on children's ILD (chILD). Data was presented on the American registry that collects data on children with a new diagnosis and prevalence cases for follow-up in management and treatment. Over the last year, there has been an increase in cases added to the registry, which 
will give important information on children with a differential diagnosis of ILD soon.

During the meeting, activity from Europe, such as the ERS Clinical Research Collaboration on ILD, the European Union chILD registry and the new COST Action on chILD were also discussed. In addition, Australia and Argentina are working on chILD registries; the Argentinian registry has a large group of patients with post-infectious bronchiolitis obliterans.

A rapid update was given by a smaller research team with interests in neuroendocrine hyperplasia of infancy, stem cell innovations and genetic research. The network is expanding its collaboration to increase awareness and to improve knowledge on chILD, in order to create and set up clinical trials for management and treatment with old or orphan drugs, or to develop new treatment options.

\section{Social media}

The ECMC wants to be able to provide all ECMs with updates on opportunities for ECMs within the ERS. Therefore, we have launched an ERS Early Career Members page on Facebook. Our other social media platforms are still up and running. Please start following us using the hyperlinks below.

- Facebook: https://www.facebook.com/groups/585753791780360

- ResearchGate: https://www.researchgate.net/project/ European-Respiratory-Society-Early-Career-Members

- Twitter: https://twitter.com/EarlyCareerERS

This interest in chILD is very high all around the world and networking is the best way to increase awareness in rare disease.

\section{Acknowledgements}

The authors' affiliations are as follows. Tiago M. Alfaro: Pneumology Unit A, Centro Hospitalar e Universitário de Coimbra, Coimbra, Portugal; and Centre of Pneumology, Faculty of Medicine, University of Coimbra, Coimbra, Portugal. Cristina Calarasu: University of Medicine and Pharmacy of Craiova, Dept of Medical Specialities, Craiova, Romania. Sheila Ramjug: Wythenshawe Hospital, Manchester Foundation Trust Hospitals, Manchester, UK. Lowie Vanfleteren: COPD Center, Sahlgrenska University, Gothenburg, Sweden. Raffaella Nenna and Laura Petrarca: Dept of Pediatrics, "Sapienza” University of Rome, Rome, Italy. Deborah Snijders: Dept of Women's and Children's Health, University of Padova, Padua, Italy. Jana De Brandt: REVAL - Rehabilitation Research Center, BIOMED - Biomedical Research Institute, Faculty of Medicine and Life Sciences, Hasselt University, Diepenbeek, Belgium.

\section{Conflict of interest}

None declared. 